\title{
Stable transfection into rat bone marrow mesenchymal stem cells by lentivirus-mediated NT-3
}

\author{
YU GONG* ${ }^{*}$, HONGFEI WANG* ${ }^{*}$ and HAIJUN XIA \\ Department of Bone Surgery, The Second Affiliated Hospital of Dalian Medical University, \\ Dalian, Liaoning 116021, P.R. China
}

Received March 20, 2014; Accepted June 5, 2014

DOI: $10.3892 / \mathrm{mmr} .2014 .2727$

\begin{abstract}
Transplantation of bone marrow mesenchymal stem cells (BMSCs) is the most promising therapeutic strategy in the treatment of spinal cord injuries. BMSCs have a wide variety of sources and are characterized by being exempt from immune rejection, marked secretory functions and neuronal plasticity during differentiation. The lentiviral vector, namely PLV.Ex3d.P/neo-EF1A-NT3-internal ribosome entry site-enhanced green fluorescent protein, was constructed and subsequently transfected into Sprague Dawley (SD) rat BMSCs. The gene and protein expression levels of the nucleic acid neurotrophin-3 (NT-3) were then detected. The results demonstrated that the constructed NT-3 gene lentiviral expression vector matched the expected design and that the NT-3 gene was transfected into the BMSCs via the lentivirus-mediated method at a transfection efficiency of $60-70 \%$. NT-3 gene expression was detected within the stably transfected positive cells at the nucleic acid and protein levels. The cell morphology and biological activity of BMSCs did not alter significantly following transfection with NT-3. NT-3-transfected SD BMSCs were successfully constructed and served as effective vector seed cells with stable expression. These results can be used as a reference for subsequent studies on the transplantation therapy of rat spinal cord injuries using lentivirus-mediated NT-3-transfected SD BMSCs.
\end{abstract}

\section{Introduction}

The clinical manifestations of acute spinal cord injuries (ASCIs) mainly appear as quadriplegia or paraplegia. In addition to primary injuries, ASCIs are also caused by secondary injuries,

Correspondence to: Dr Hongfei Wang, Department of Bone Surgery, The Second Affiliated Hospital of Dalian Medical University, 467 Zhongshan Road, Dalian, Liaoning 116021, P.R. China

E-mail: hongfeiwangcn@126.com

*Contributed equally

Key words: lentivirus NT-3, transfection, mesenchymal stem cell, gene construction including ischemia, hypoxia, the inflammatory response, free radical formation and excitotoxicity, which could take several hours or days to manifest following the primary injuries. These secondary injuries can in turn result in the formation of severe spinal cord injuries (SCIs), for which no effective treatment is available to date (1). Stem cell transplantation is currently the most promising treatment method for SCIs. In particular, bone marrow mesenchymal stem cells (BMSCs) are considered highly promising in SCI clinical therapy and cell transplantation $(2,3)$. In addition to their wide availability and relative ease of isolation and expansion, BMSCs are able to differentiate into neurons (4). However, transplanting BMSCs into the SCI site and inducing them to differentiate into neurons remains a challenge and has yielded a number of inconsistent results in animal models $(5,6)$. These results were associated with the deficiency of neurotrophic factors in the local microenvironment during SCI repair. Therefore, the importance of improving the local neurotrophic factor levels and creating a gentle microenvironment for the damaged spinal cord sites has become apparent in the study of SCIs.

Neurotrophin-3 (NT-3), a member of the neurotrophin family, is involved in the neuroprotection and nutrition of various neurons and thus promotes the survival and axonal regeneration of damaged neurons (7). However, due to its relatively short half-life (8) and its rapid metabolism when combined with BMSCs, NT-3 cannot sustain its effects on SCIs. In order to address this problem, a combined treatment involving the transfection of neurotrophic factor genes into BMSC cells was designed. This combination allows the sustained secretion of neurotrophic factors, improves the survival of BMSCs and is involved in induced neuronal differentiation in order to maximize the therapeutic effects in SCIs. The current study aimed to transfect the NT-3 gene in vitro into cultured Sprague Dawley (SD) rat BMSCs using a lentivirus-mediated method in order to determine the efficiency of NT-3 gene-BMSC transfection and NT-3 gene expression in BMSCs.

\section{Materials and methods}

Plasmid construction. The attB1-NT3-attB2 sequence was amplified using the overlapping polymerase chain reaction (PCR) method. The primers used were as follows: attB1-Kozak-NT3-P, forward 5-'ATG TCC ATC TTG TTT TAT GTG ATA TTT C-3' and reverse 5'-TCA TGT TCT TCC GAT TTT TCT TGA 
C-3'. The PCR products were then subjected to $1 \%$ agarose gel electrophoresis. Subsequently, the DNA was recycled with the agarose gel electrophoresis recycling kit, according to the manufacturer's instructions (Qiagen Inc., Valencia, CA, USA) and stored at $-20^{\circ} \mathrm{C}$.

Gateway cloning was performed to construct the entry clone. pDown-NT3 was added to the pDonr221 plasmid, BP clonase and attB1-NT3-attB2. The mixture was incubated at $25^{\circ} \mathrm{C}$ and was then reacted with BP for $3 \mathrm{~h}$. Proteinase K (Cyagen Biosciences Inc., Guagzhou, China) was added for $10 \mathrm{~min}$ to terminate the reaction. Subsequently, the BP reaction products were transferred into the Escherichia coli strain DH5 $\alpha$ (Cyagen Biosciences Inc.).Following this, $100 \mu \mathrm{l}$ of the transformed product was coated onto a lysogeny broth plate with $50 \mu \mathrm{g} / \mathrm{ml}$ Kan and subsequently incubated overnight at $37^{\circ} \mathrm{C}$. Colony PCR was used to screen the positive plasmid clones, which were then used for positive clone sequencing.

Gateway cloning was performed to construct the recombinant target plasmid, PLV.Ex3d.P-neo-EF1A-NT3-internal ribosome entry site (IRES)-enhanced green fluorescent protein (EGFP). Minor extractions of the pUp-EF1A, pDown-NT3, pTail-IRES-EGFP, LR clonase and PLV.Des3d.P-neo (Tiangen Biotech (Beijing) Co., Ltd., Beijing, China) plasmids were performed, and the extracts were incubated at $25^{\circ} \mathrm{C}$. Subsequently, the incubated samples were subjected to LR reaction for $3 \mathrm{~h}$. Proteinase $\mathrm{K}$ was added for $10 \mathrm{~min}$ to terminate the reaction. The LR reaction products were then transferred into the $E$. coli strain DH5 $\alpha$. Colony PCR was used to screen the positive plasmid clones, which were then used for positive clone sequencing.

Lentivirus packaging. 293FT cells (Cyagen Biosciences Inc.) in the logarithmic growth phase and in good condition were obtained. Following digestion and counting, the cells were seeded in a $10 \mathrm{~cm}$ dish at a concentration of $5 \times 10^{6}$ cells $/$ dish and then incubated overnight at $37^{\circ} \mathrm{C}$ and $5 \% \mathrm{CO}_{2}$. Prior to transfection, the used culture solution was removed and $5 \mathrm{ml}$ of $10 \%$ fresh Dulbecco's modified Eagle's medium was added to prepare a DNA-Lipofectamine 2000 complex (Invitrogen Life Technologies, Carlsbad, CA, USA). The quantity of complex in the $10 \mathrm{~cm}$ dish was used as a reference. The DNA-Lipofectamine 2000 complex was added dropwise onto the 293FT cells and the dish was agitated to achieve mixing. Subsequently, the mixture was incubated overnight at $37^{\circ} \mathrm{C}$ in a $5 \% \mathrm{CO}_{2}$-humidified incubator (NuAire, Inc., Plymouth, $\mathrm{MN}$, USA). Approximately $48 \mathrm{~h}$ after the transfection, the cell supernatant of the transfected 293FT cells (in the logarithmic growth phase) was collected and concentrated, and then was added to a $10 \mathrm{ml}$ culture dish for continuous cultivation. After $72 \mathrm{~h}$, the cell supernatant was collected and reconcentrated and the virus was subpacked. The sample was then stored at $-80^{\circ} \mathrm{C}$ for use in the determination of the biological virus titer. The virus titers were preliminarily determined according to their fluorescent expression.

Cultivation, passage and lentiviral-stable transfection of SD rat BMSCs. An SD rat was sacrificed via cervical dislocation. The whole body was then immersed in $75 \%$ ethanol for $30 \mathrm{~min}$. The bilateral femur and tibia were obtained under sterile conditions and washed with the medium. Subsequently, the samples were prepared into single-cell suspensions. The cell concentration was adjusted and the sample was placed in an incubator at $37^{\circ} \mathrm{C}$. Cell passage was performed when $80-90 \%$ of the cells were fused. The present study was conducted in strict accordance with the recommendations in the Guide for the Care and Use of Laboratory Animals of the National Institutes of Health (Bethesda, MD, USA). The animal use protocol has been reviewed and approved by the Institutional Animal Care and Use Committee of the Second Affiliated Hospital of Dalian Medical University (Dalian, China).

Fifth-generation BMSCs were obtained for digestion and were centrifuged at $168 \mathrm{x} \mathrm{g}$, for $5 \mathrm{~min}$. Subsequently, the supernatant was discarded and the cell precipitate was resuspended. Several samples were obtained for counting and the cells were seeded into a six-well plate at a density of $1 \times 10^{5}$ cells $/ 9.6 \mathrm{~cm}^{2}$. When the cells had completely adhered to the wall, the medium was replaced with $1 \mathrm{ml}$ of a fresh SD MSC complete medium (Gibco-BRL, Carlsbad, CA, USA). The cells in two wells were then added with 40 and $60 \mu 1$ NT-3 and EGFP-carrying lentivirus, respectively. Following complete mixing by agitation, the samples were incubated at $37^{\circ} \mathrm{C}, 5 \% \mathrm{CO}_{2}$ and $95 \%$ relative humidity. Approximately $8 \mathrm{~h}$ after the transduction, the SD BMSC complete medium was replaced and transduction was continued for 48 h. Following this, transduction results for the samples were determined using an inverted fluorescence microscope (Olympus, Tokyo, Japan). Overgrown cells were inoculated onto a six-well plate for drug screening using two 0.5 and $1.0 \mathrm{mg} / \mathrm{ml} \mathrm{G} 418$ solutions (Shanghai Fushen Biotechnology Co., Ltd., Shanghai, China). The medium was replaced every 2 days. The expression of green fluorescent protein (GFP) was observed using inverted fluorescence microscopy based on the color of the medium and the cell state. Following 7 days of screening, the complete medium without G418 was replaced and cultivation was continued.

Quantitative PCR ( $q P C R)$. RNA was extracted and its concentration and purity were determined using an ultra microspectrophotometer (Thermo Fisher Scientific, Inc., Waltham, MA, USA). Following reverse transcription, the synthesized cDNA samples were subjected to qPCR (Applied Biosystems, Foster City, CA, USA) using the following primer sequences: NT3, forward 5'-ACC AGGTTA CAG TGT TGG GAG AG-3' and reverse 5'-CGC TGA GAG TTC CAG TGT TTG TC-3'; rat GAPDH, forward 5'-CCT TCC GTG TTC CTA CCC-3' and reverse 5'-CAA CCT GGT CCT CAG TGT AG-3'. The PCR reaction conditions were as follows: predenaturation, $95^{\circ} \mathrm{C}$ for $60 \mathrm{sec}$; PCR cycles (x40) consisting of $95^{\circ} \mathrm{C}$ for $15 \mathrm{sec}$ and then $60^{\circ} \mathrm{C}$ for $1 \mathrm{~min}$; melting curve analysis at $95^{\circ} \mathrm{C}$ for $15 \mathrm{sec}(100 \%), 70^{\circ} \mathrm{C}$ for $1 \mathrm{~min}(1 \%)$ and at $95^{\circ} \mathrm{C}$ for $30 \mathrm{sec}$.

Western blotting. Radioimmunoprecipitation assay lysate (Beyotime Institute of Biotechnology, Shanghai, China) was used to extract the total cellular proteins. A nucleic acid protein analyzer (Thermo Fisher Scientific, Inc.) was then used to determine the total concentrations of the sample proteins. Following SDS-polyacrylamide gel electrophoresis, the proteins were transferred and hybridized. The membrane was incubated overnight with the appropriately diluted 
primary antibodies (rabbit anti-rat NT-3 polyclonal antibody; Sigma-Aldrich, St. Louis, MO, USA) at $4^{\circ} \mathrm{C}$. Subsequently, the membrane was incubated with the appropriately diluted secondary antibodies (Abcam, Cambridge, MA, USA) at room temperature for $1 \mathrm{~h}$ and stained using an enhanced chemiluminescence kit (Thermo Fisher Scientific, Inc.). The membrane was analyzed using a Fiuorchem HD2 gel-imaging analysis system (ProteinSimple Inc., Santa Clara, CA, USA), and the development and fixation of specific protein bands were detected.

Observation indicators. Electrophoretic identification and sequencing identification of the recombinant plasmid PLV. Ex3d.P/neo-EF1A >NT3>IRES/EGFP was performed. Following the lentiviral packaging, the expression of GFP and the positive transfection efficiency were determined. The mRNA and protein expression in the PLV. Ex3d.P/neo-EF1A/NT3/IRES/EGFP-transfected BMSCs was determined.

\section{Results}

Electrophoresis of the PCR products of the SD rat recombinant NT-3 gene. PCR amplification yielded the $\sim 841$ bp NT-3 gene fragment attB1-NT3-attB2 (Fig. 1A). Gateway clone technology was used to generate the entry clone pDown-NT3 ( 1041 bp; Fig. 1B) via the BP reaction and to produce the recombinant target plasmid PLV. Ex3d.P-neo-EF1A/NT3/-IRES/EGFP ( 830 bp; Fig. 1C). The colony was screened for positive clones. Sequencing of the recombinant target plasmid demonstrated that the expected sequence was achieved. This result indicated the successful construction of the recombinant target plasmid PLV.Ex3d.P-neo-EF1A-NT3-IRES-EGFP.

Results of the lentiviral packaging. A lentiviral packaging system was used to pack the 293FT cells in the logarithmic growth phase, as shown in the fluorescence image (Fig. 2A). The fluorescence image of pLV.Ex2d.P/neo-EF1A/EGFP in the control group (Fig. 2B) shows the generation of EGFP fluorescence proteins. This result indicated the successful packaging of the lentivirus.

Determination of the fluorescence expression of the lentivirus titer. The number of fluorescent cells decreased with increasing dilution. At a stock lentivirus solution of $10^{-5} \mu \mathrm{l}$, the GFP remained expressed. Thus, the preliminary lentivirus titer was determined as $3.5 \times 10^{-8} \mathrm{TU} / \mathrm{ml}$.

Establishment of stably transfected SD BMSCs. The PLV Ex 3d.P-neo-EF1A-NT3-IRES-EGFP-transfected BMSCs were purified via drug screening and observed under an inverted fluorescence microscope. The results demonstrated the growth of a positive cell colony. Following the transfection, no significant change was identified in the BMSC morphology and the cells uniformly expressed GFP. The transfection efficiency was 60-70\% (Fig. 3)

Determination of NT-3 expression. Western blotting and qPCR were mainly used to verify the transcription and

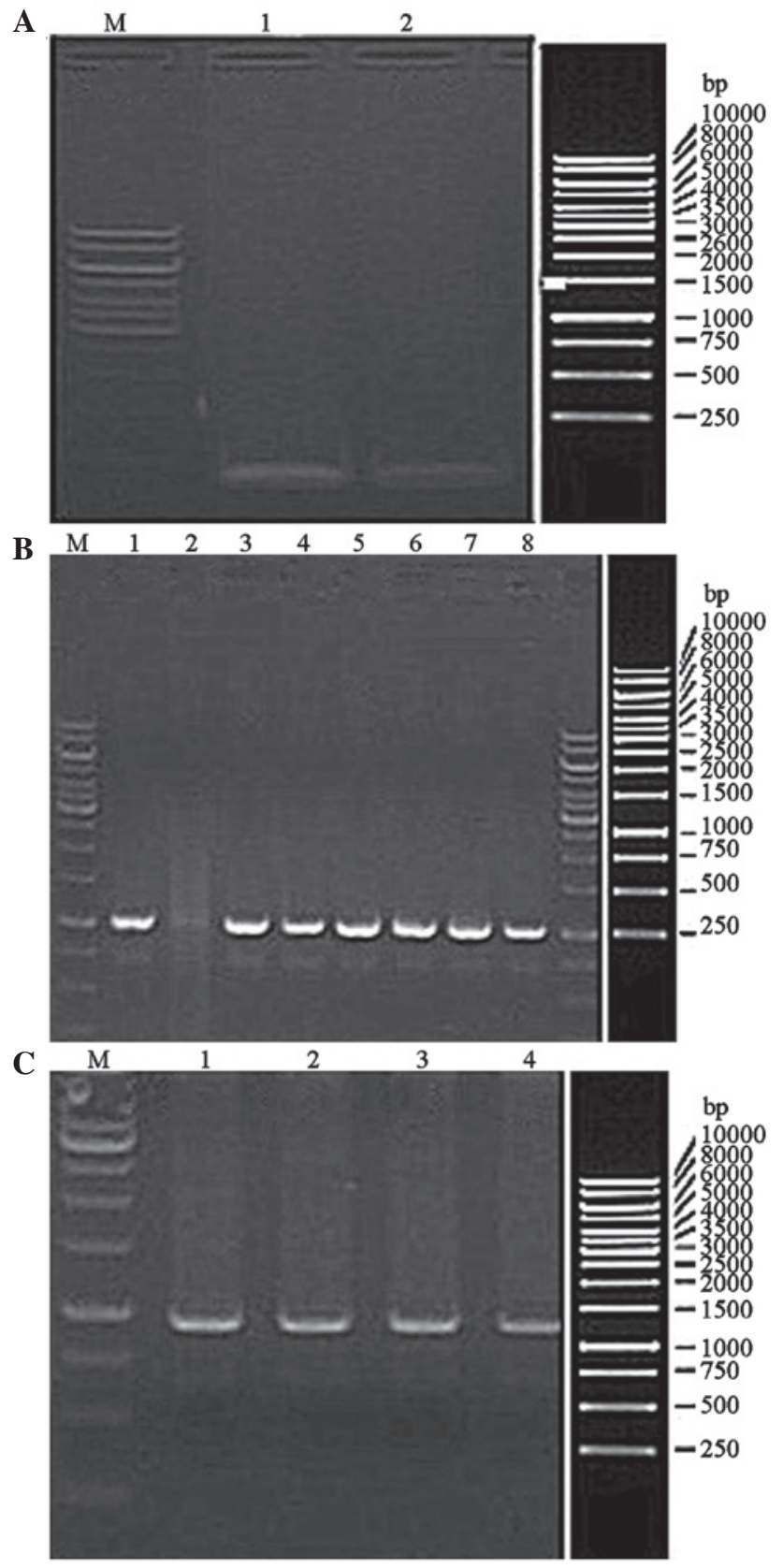

Figure 1. (A) Lanes 1-2: attB1-NT3-attB2 polymerase chain reaction products; (B) Lanes 1-8: pDown-NT3 clone No. 1-8; (C) Lanes 1-4: PLV. Ex3d.P/neo-EF1A/NT3/internal ribosome entry site/enhanced green fluorescent protein clone No. 1-4. BP, base pairs; M, marker; NT-3, neurotrophin-3.

translation of NT-3 in the transfected cells, particularly in terms of nucleic acid and protein levels. The mRNA levels in the NT-3-transfected BMSCs significantly increased (Fig. 4) compared with those of the untransfected group. Similar results were obtained from the western blot analysis. The NT-3 protein band in the untransfected BMSC group was significantly weaker than that in the transfected group (Fig. 5). These results indicated that the NT-3 protein expression in the NT-3-transfected BMSC group was significantly higher than that in the untransfected group.

The findings of the present study demonstrated that the Gateway clone technique successfully constructed the recombinant target plasmid PLV.Ex3d.P-neo-EF1A-NT3-IRES-EGFP 
A

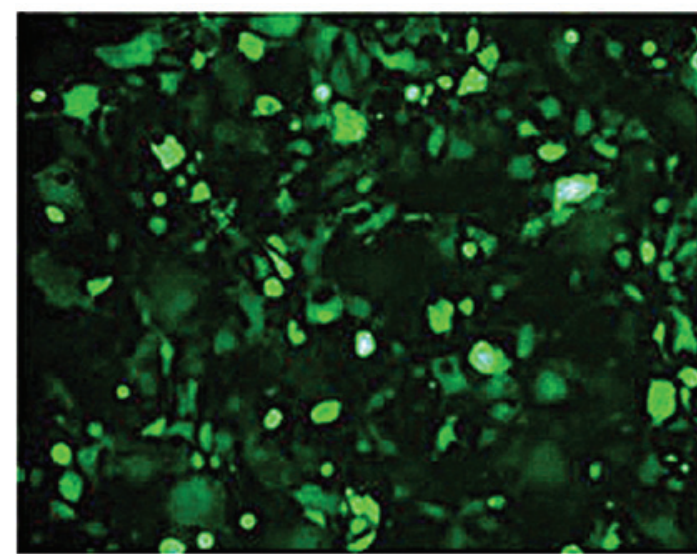

B

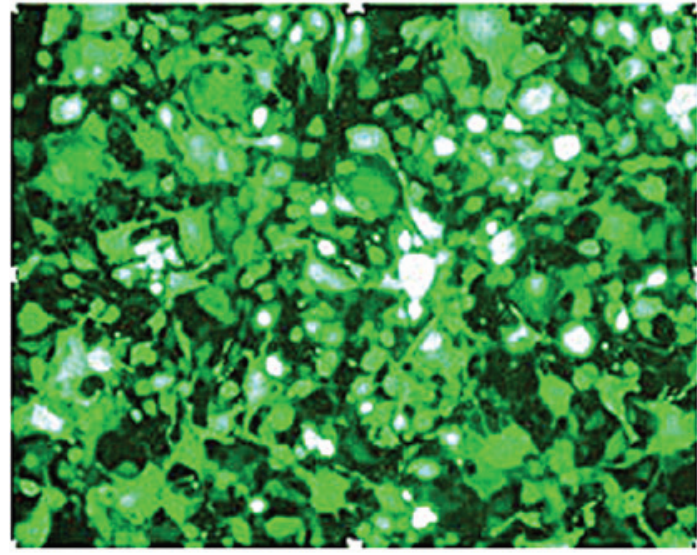

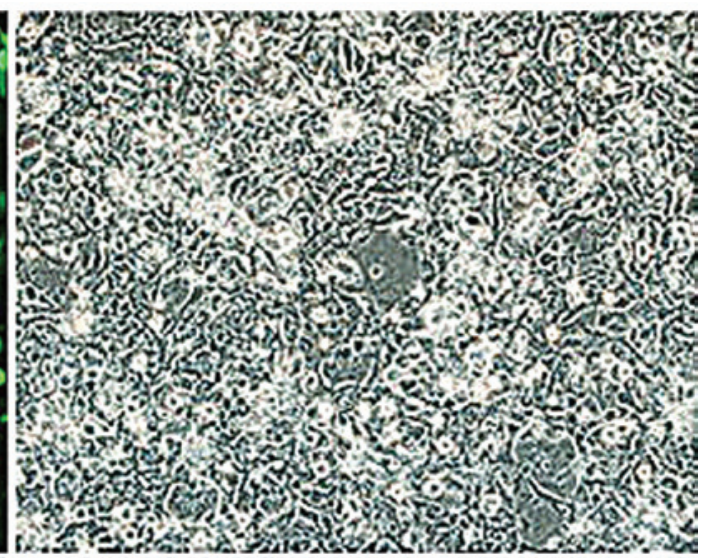

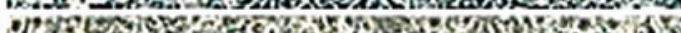

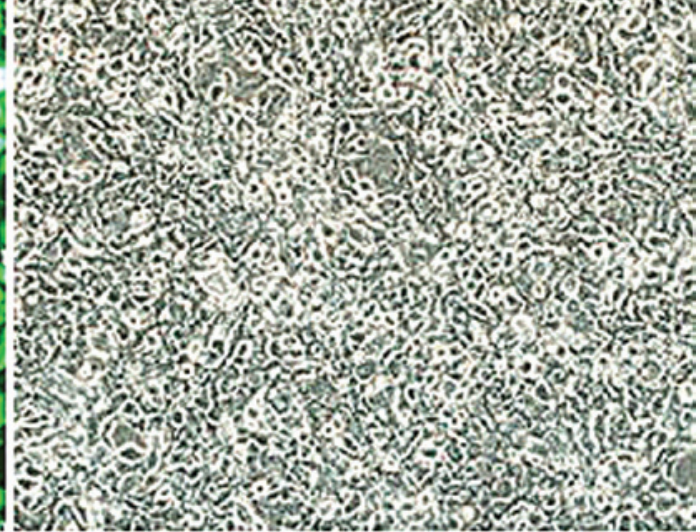

Figure 2. Lentivirus package. (A) Cell fluorescence images $48 \mathrm{~h}$ after the transfection of PLV.Ex3d.P-neo-EF1A-NT3-internal ribosome entry site-EGFP into 293FT (magnification, x200); (B) Cell fluorescence images $48 \mathrm{~h}$ after the PLV.Ex2d.P/neo-EF1A/EGFP transfection into 293FT (magnification, x200). EGFP, enhanced green fluorescent protein.

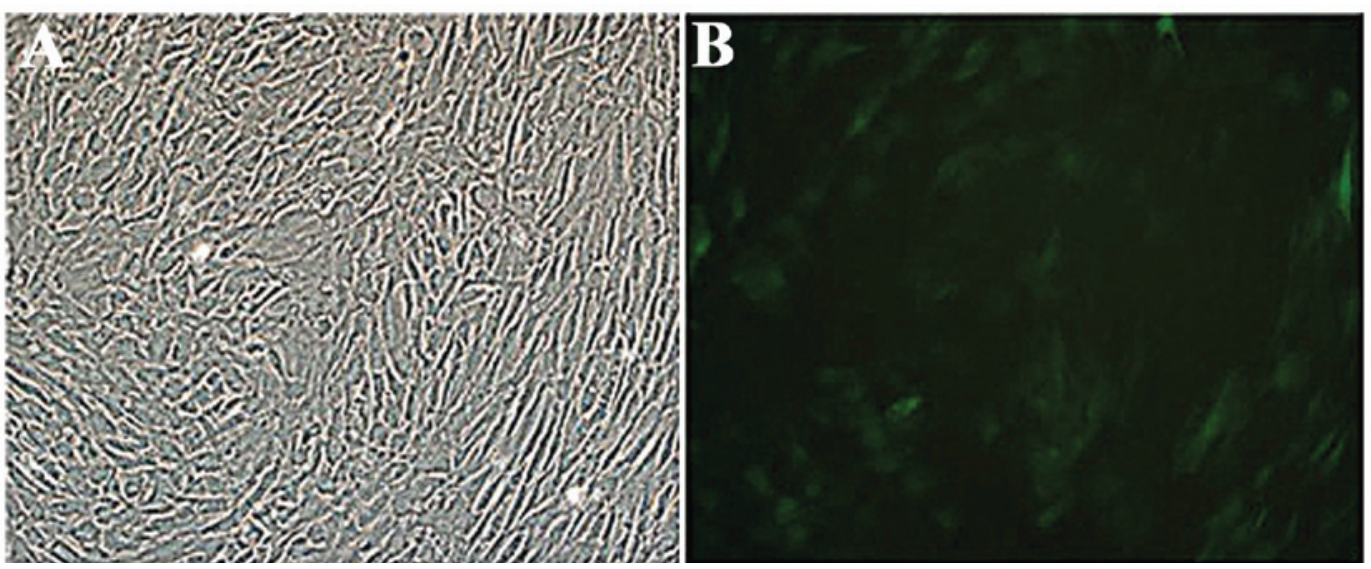

Figure 3. Cell fluorescence images $48 \mathrm{~h}$ after the PLV.Ex3d.P-neo-EF1A-NT3-internal ribosome entry site-enhanced green fluorescent protein transfection into bone marrow mesenchymal stem cells (magnification, x200). (A) Lentiviral transduction following complete cell adherence; (B) $48 \mathrm{~h}$ post-transduction, the cells were uniform, with green fluorescence. The transduction rate was $60-70 \%$.

and efficiently transfected it into the SD rat BMSCs, which maintained a high NT-3 expression for a long period.

\section{Discussion}

In SCI repair, deficiency in neurotrophic factors in the damaged local microenvironment is generally considered an important factor that determines functional recovery following SCI (9). Therefore, increasing the concentration of neurotrophic factors in the microenvironment may promote and induce the regeneration of nerve cells and is a potential therapeutic treatment strategy for SCIs. Zhou et al (10) confirmed that NT-3 has a significant role in inhibiting neuronal apoptosis and promoting axonal regeneration during SCI repair and is considered the most effective of the neurotrophic factors. However, the topical application of NT-3 in SCIs is clinically limited due to its short half-life, immunogenicity, difficulty in passing through the blood/spinal barrier and ineffective promotion in 
A

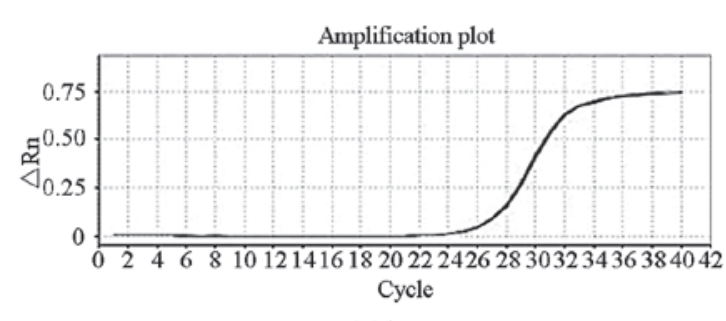

Melt curve

C
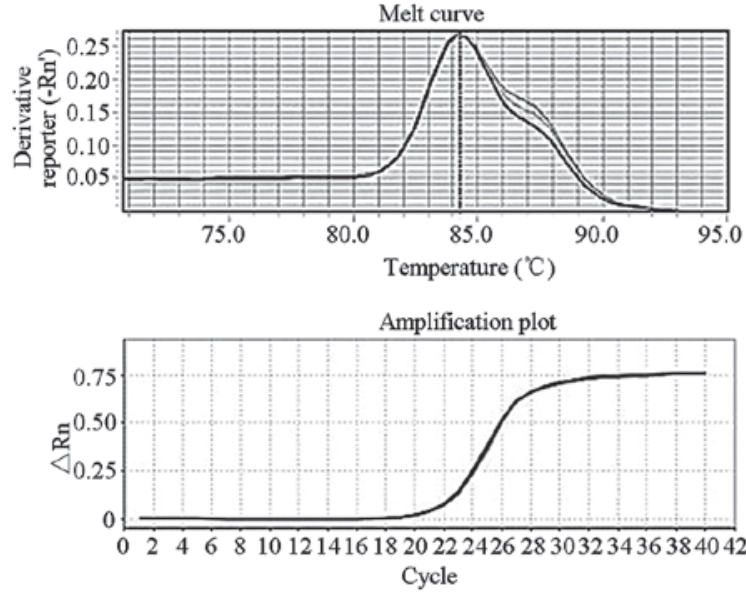

Melt curve

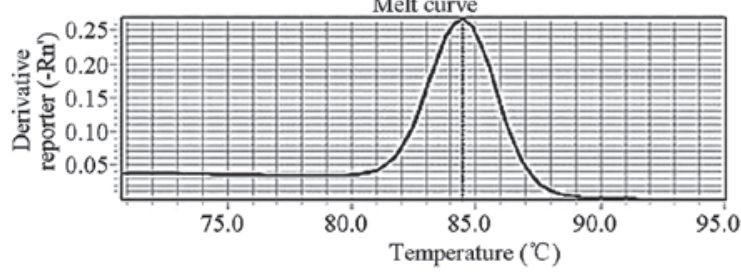

B

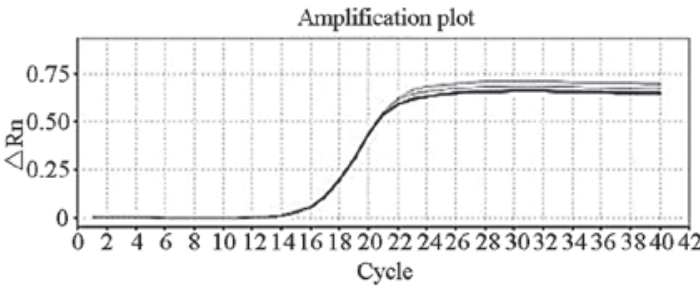

Melt curve

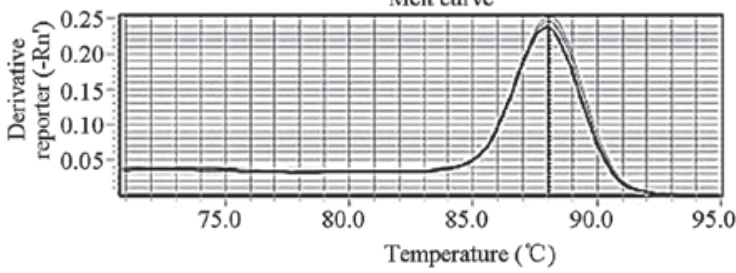

D

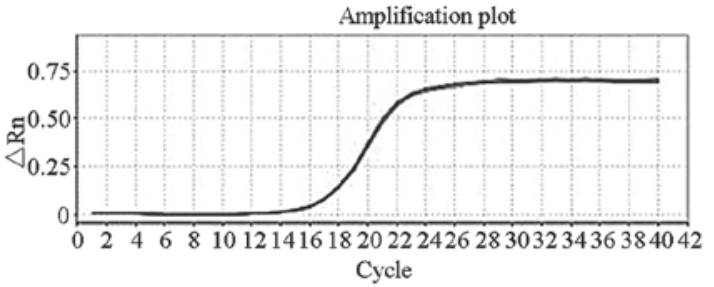

Melt curve

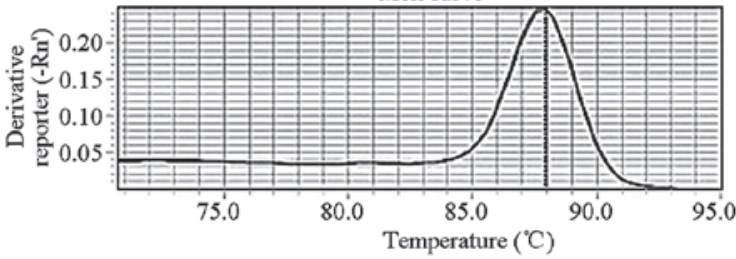

Figure 4. Expression of the nt-3 gene in lentivirus stable transfected mesenchymal stem cells detected by quantitative polymerase chain reaction. (A) Amplification plot and melt curve of the NT-3 gene in SD BMSCs; (B) Amplification plot and melt curve of GAPDH gene; (C) Amplification plot and melt curve of the NT-3 gene in SD BMSCs/NT-3; (D) Amplification plot and melt curve of GAPDH gene. SD, Sprague Dawley; BMSCs, bone marrow mesenchymal stem cells; NT-3, neurotrophin-3.

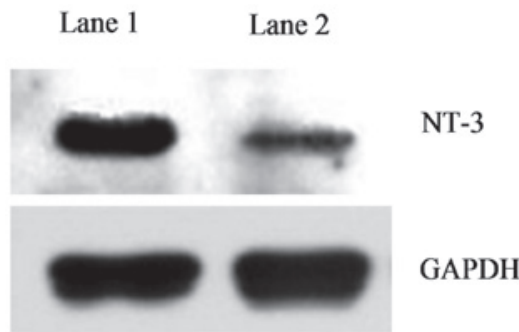

Figure 5. Expression of the NT-3 gene in lentivirus stable transfected mesenchymal stem cells detected by western blotting. Lane 1, SD BMSCs/NT3; Lane 2, SD BMSCs. BMSC, bone marrow mesenchymal stem cells, SD, Sprague Dawley; NT-3, neurotrophin-3.

spinal pathway reconstruction following syringomyelia. To overcome these disadvantages, molecular biological methods were designed to transfer the therapeutic NT-3 gene into a suitable cell vector and allow the gene to maintain a stable expression in the cell. The NT-3 gene-carrying cell vector was then replanted in the SCIs to achieve sustained secretion and thus promote the recovery of neurological functions following SCI. To a certain extent, these methods overcome the limitations of NT-3 in SCI treatment.

Neurotrophic factors and their receptors are widely present in the spinal cord. However, with the exception of the high
NT-3 levels during early spinal cord development (11), the levels of other neutrophic factors are relatively low in the spinal cord. Among the receptor tyrosine kinases, tyrosine kinase receptor C has a marked affinity for NT-3 (12). As an important member of neurotrophic factor families, NT-3 is involved in the development and differentiation of neurons and can promote the survival of damaged central neurons as well as the regeneration of nerve fibers (13). In addition, NT-3 promotes the proliferation of oligodendrocytes and the myelination of regenerated axons and serves as a guide for damaged axons (14).

BMSCs are fibroblast-colony units derived from the bone marrow. These cells exhibit a high degree of self-renewal capacity, marked proliferative capacity, high plasticity and potential pluripotency (15). Furthermore, BMSCs self-originate and thus have low immunogenicity, are easily sourced and can stably express a variety of exogenous target genes in vivo and in vitro (16). Due to these features, BMSCs have been used in the cell therapy of various diseases and as vector cells in gene therapy (17) Brazelton et al (18) and Mezey et al (19) found that in vivo, exogenous BMSCs differentiate into glial cells and neurons via different routes following migration into the mouse brain. This finding suggests the feasibility of using transplanted BMSCs to treat central nervous system damage. Hofstetter et al (20) transplanted BMSCs to treat a standard 
spinal cord injury in a rat model and demonstrated that BMSCs can promote the recovery of hind limb functions. Several studies attributed this neuroprotective effect of BMSCs to their ability to differentiate into neurons, which replace diseased or damaged neurons (21). However, Mahmood et al (22) attributed the ability of transplanted BMSCs to promote functional recovery in SCIs to the secretion of neurotrophic factors by these cells within the damaged tissues. These neurotrophic factors in turn promote neuronal survival and induce the proliferation of endogenous cells and the regeneration of nerve fibers. Further studies demonstrated that BMSCs have high biocompatibility as well as excellent immunoregulation and immune circumvention abilities. Therefore, BMSCs have become the dominant vectors of neurotrophin cells (23). In the quantitative analysis of neurotrophic factors secreted by BMSCs, Crigler et al (24) detected the expression of neurotrophic factors, including brain-derived neurotrophic factor and nerve growth factor but did not detect NT-3 expression.

In the present study, the lentivirus-mediated method was used to transfect the NT-3 gene into SD rat BMSCs in order to establish NT-3-carrying BMSC strains. Gateway clone technology was used in the recombination and construction of the target plasmid. This technique is a new universal vector construction method based on the phage $\lambda$ site-specific recombination system (25), which can rapidly clone heterologous DNA fragments into different vectors with high specificity. The recombination sites include attB, attP, attL and attR. The recombination reactions include the $\mathrm{BP}$ recombination reaction, in which attP-containing donor vectors react with attB-containing PCR products, sequence-exchange reactions occur at the att sites and entry clones are generated and the LR recombination reaction, in which attL-containing entry clones react with attR-containing target vector, a sequence-exchange reaction occurs at the att sites and eukaryotic expression vectors of the target gene are generated. Compared with traditional vector construction methods, including restricted enzyme digestion and ligation, Gateway cloning is easy and highly efficient. Furthermore, this method can significantly reduce false positive results during vector construction (26), keep the developing and reading frames unchanged and achieve fast and efficient transfers of DNA fragments (27). In the present study, the overlapping PCR amplification method was used to obtain attB1-NT-3-attB2. Gateway cloning then successfully constructed the recombinant target plasmid PLV. Ex3d.P-neo-EF1A-NT3-IRES-EGFP for the viral packaging. Although adenoviral vectors have high transfection rates, their corresponding immune response poses certain risks in in vivo experiments (28). Therefore, a lentivirus was used as the expression vector to prevent unwanted side effects. A third-generation lentiviral packaging system was used to pack the $293 \mathrm{FT}$ cells into the viral particles, which were then transfected with Lipofectamine 2000. The fluorescence image demonstrated that EGFP was generated at a transfection rate $>90 \%$. The lentiviral vector, which completed the viral packaging and carried NT-3/EGFP, was transfected into the SD BMSCs. NT-3/EGFP-carrying SD BMSCs strains were obtained following drug screening and purification. qPCR and western blotting confirmed that the NT-3 gene transfected into SD BMSCs maintained a stable expression. The recombinant lentiviral vector significantly improved the gene transfection efficiency of the BMSCs and the probability of target gene integration into the host cell genome was significantly increased. The efficient integration of a foreign gene into the host chromosome resulted in a stable and lasting expression (29). The BMSCs stably and efficiently expressed the foreign genes and thus overcame the disadvantages of topical cytokine application.

In conclusion, the present study used a recombinant lentiviral vector as the expression vector to transfect the target gene PLV Ex3d.P/neo-EF1A-NT3-IRES-EGFP into the SD rat BMSC genome. The transfected BMSCs successfully expressed NT-3 and EGFP. In addition, the transfected BMSCs exhibited a stable NT-3 secretion and the EGFP gene successfully traced and located the site of target gene expression in vivo and in vitro. The results of the present study provide a solid foundation for further experimental studies on NT-3 gene-modified BMSC transplantation for SCI treatment. The results may also serve as a reference for the development of reliable NT-3 gene-modified BMSCs.

\section{Acknowledgements}

This study was supported by the Liaoning Provincial Science and Technology Project Foundation (grant no. 2011225013).

\section{References}

1. Sahani V and Kessler JA: Stem cell therapies for spinal cord injury. Nat Rev Neurol 6: 363-372, 2010.

2. Sensebe L, Krampera M, Schrezenmeier H, Bourin P and Giordano R: Mesenchymal stem cell for clinical application. Vox Sang 98: 93-107, 2010.

3. Uccelli A, Moretta L and Pistoia V: Mesenchyal stem cells in health and disease. Nat Rev Immunol 8: 726-736, 2008.

4. Sanchez-Ramos J, Song S, Cardozo-Pelaez F, et al: Adult bone marrow stromal cells differentiate into neural cells in vitro. Exp Neurol 164: 247-256, 2000.

5. Lu P, Jones LL and Tuszynski MH: BDNF-expressing marrow stromal cells support extensive axonal growth at sites of spinal cord injury. Exp Neurol 191: 344-360, 2005.

6. Ohta M, Suzuki Y, Noda T, et al: Bone marrow stromal cells infused into the cerebrospinal fluid promote functional recovery of the injured rat spinal cord with reduced cavity formation. Exp Neurol 187: 266-278, 2004.

7. Tuszynski MH, Grill R, Leonard LL, et al: NT-3 gene delivery elicits growth of chronically injured corticospinal axons and modestly improves functional deficits after chronic scar resection. Exp Neurol 181: 47-56, 2003.

8. Ejstrup R, Kiilgaard JF, Tucker BA, Klassen HJ, Young MJ and La Cour M: Pharmacokinetics of intravitreal glial cell line-derived neurotrophic factor: experimental studies in pigs. Exp Eye Res 91: 890-895, 2010.

9. Fawectt J: Repair of spinal cord injuries: where are we, where are we going? Spinal Cord 40: 615-623, 2002.

10. Zhou L, Baumgartner BJ, Hill-Felberg SJ, McGowen LR and Shine HD: Neurotrophin-3 expressed in situ induces axonal plasticity in the adult injured spinal cord. J Neurosci 23: 1424-1431, 2003.

11. Zhang W, Li Y, Wang ZJ, Zhou X, Ou KQ, Zhou HL and Wang TH: Functional roles of intrinsic neurotrophin-3 in spinal neuroplasticity of cats following partial ganglionectomy. Growth Factors 28: 351-358, 2010.

12. Ba YC, Dai P, Zhou HL, Liu J and Wang TH: Spatiotemporal changes of NGF, BDNF and NT-3 in the developing spinal cords of embryonic chicken. Neurochem Res 35: 273-278, 2010.

13. Chapleau CA and Pozzo-Miller L: Divergent roles of p75NTR and Trk receptors in BDNF's effects on dendritic spine density and morphology. Neural Plast 2012: 578057, 2012.

14. Xian HQ, McNichols E, St Clair A and Gottlieb DI: A subset of ES-cell-derived neural cells marked by gene targeting. Stem Cells 21: 41-49, 2003. 
15. Wang XJ and Li QP: The roles of mesenchymal stem cells (MSCs) therapy in ischemic heart diseases. Biochem Biophys Res Commun 359: 189-193, 2007.

16. Lu L, Zhao C, Liu Y, et al: Therapeutic benefit of TH-engineered mesenchymal stem cells for Parkinson's disease. Brain Res Brain Res Protoc 15: 46-51, 2005.

17. Kan EM, Ling EA and Lu J: Stem cell therapy for spinal cord injury. Curr Med Chem 17: 4492-4510, 2010

18. Brazelton TR, Rossi FM, Keshet GI and Blau HM: From marrow to brain: expression of neuronal phenotype in adult mice. Science 290: 1775-1779, 2000.

19. Mezey E, Chandross KJ, Harta G, Maki RA and McKercher SR: Turning blood into brain: cells bearing neuronal antigens generated in vivo from bone marrow. Science 290: 1779-1782, 2000.

20. Hofstetter CP, Schwarz EJ, Hess D, Widenfalk J, El Manira A, Prockop DJ and Olson L: Marrow stromal cells form guiding strands in the injured spinal cord and promote recovery. Proc Natl Acad Sci USA 99: 2199-2204, 2002.

21. Black IB and Woodbury D: Adult rat and human bone marrow stromal stem cells differentiate into neurons. Blood Cells Mol Dis 27: 632-636, 2001.

22. Mahmood A, Lu D and Chopp M: Marrow stroma cell transplantation after traumatic brain injury promotes cllular proliferation within the brain. Neurosurgery 55: 1185-1193, 2004.
23. Wang Y, Liu J, Xu C, et al: Bone marrow transplantation combined with mesenchymal stem cells induces immune tolerance without cytotoxic conditioning. J Surg Res 171: e123-e131, 2011.

24. Crigler L, Robey RC, Asawachaicham A, Gaupp D and Phinney DG: Human mesenchymal stem cell subpopulations express a variety of neuro-regulatory molecules and promote neuronal cell survival and neuritogenesis. Exp Neurol 198: 54-64, 2006.

25. Landy A: Dynamic, structural, and regulatory aspects of lambda site-specific recombination. Annu Rev Biochem 58: 913-949, 1989.

26. Bernard $\mathrm{P}$ and Couturier M: Cell killing by the F plasmid CcdB protein involves poisoning of DNA-topoisomerase II complexes. J Mol Biol 226: 735-745, 1992.

27. Magnani E, Bartling L and Hake S: From Gateway to MultiSite Gateway in one recombination event. BMC Mol Biol 7: 46, 2006.

28. Su JG, Zhu ZY, Wang Y, Xiong F and Zou J: The cytomegalovirus promoter-driven short hairpin RNA constructs mediate effective RNA interference in zebrafish in vivo. Mar Biotechnol 10: 262-269, 2008.

29. Fan JH and Li YJ: Cloning and sequence analysis of the M gene of porcine epidemic diarrhea virus LJB/03. Virus Genes 30: 69-73, 2005. 